\title{
AICAR inhibits ceramide biosynthesis in skeletal muscle
}

\author{
Katherine A Erickson ${ }^{1}$, Melissa E Smith ${ }^{1}$, Tamil S Anthonymuthu², Michael J Evanson ${ }^{1}$, Eric S Brassfield ${ }^{1}$, \\ Aimee E Hodson', M Andrew Bressler', Braden J Tucker ${ }^{1}$, Mikayla O Thatcher ${ }^{1}$, John T Prince ${ }^{2}$, \\ Chad R Hancock ${ }^{3}$ and Benjamin T Bikman ${ }^{1 *}$
}

\begin{abstract}
Background: The worldwide prevalence of obesity has lead to increased efforts to find therapies to treat obesity-related pathologies. Ceramide is a well-established mediator of several health problems that arise from adipose tissue expansion. The purpose of this study was to determine whether AICAR, an AMPK-activating drug, selectively reduces skeletal muscle ceramide synthesis.

Methods: Murine myotubes and rats were challenged with palmitate and high-fat diet, respectively, to induce ceramide accrual, in the absence or presence of AICAR. Transcript levels of the rate-limiting enzyme in ceramide biosynthesis, serine palmitoyltransferase 2 (SPT2) were measured, in addition to lipid analysis. Student's $t$-test and ANOVA were used to assess the association between outcomes and groups.

Results: Palmitate alone induced an increase in serine palmitoyltransferase 2 (SPT2) expression and an elevation of ceramide levels in myotubes. Co-incubation with palmitate and AICAR prevented both effects. However, ceramide and SPT2 increased with the addition of compound C, an AMPK inhibitor. In rats fed a high-fat diet (HFD), soleus SPT2 expression increased compared with normal chow-fed littermates. Moreover, rats on HFD that received daily AICAR injections had lower SPT2 levels and reduced muscle ceramide content compared with those on HFD only.

Conclusions: These results suggest that AICAR reduces ceramide synthesis by targeting SPT2 transcription, likely via AMPK activation as AMPK inhibition prevented the AICAR-induced improvements. Given the role of skeletal muscle ceramide in insulin resistance, it is tempting to speculate that interventions that activate AMPK may lead to long-term ceramide reduction and improved metabolic function.
\end{abstract}

Keywords: AICAR, Ceramide, AMPK, Obesity, Metabolic syndrome

\section{Background}

Obesity predisposes individuals to a host of health complications, including insulin resistance, hypertension, and dyslipidemia, which characterize the metabolic syndrome [1]. Due to the massive and sustained increase in obesity and the prevalence of the metabolic syndrome worldwide in recent decades [2], extensive efforts have been devoted to better understand the cellular events that mediate obesityrelated pathologies.

Long known to regulate apoptotic signals [3], the biologically active sphingolipid ceramide is more recently

\footnotetext{
* Correspondence: benjamin_bikman@byu.edu

'Physiology and Developmental Biology, Brigham Young University, 593 WIDB, Provo, UT 84602, USA

Full list of author information is available at the end of the article
}

known to mediate several of the deleterious conditions associated with obesity, such as insulin resistance, hepatic steatosis, and cardiovascular complications [4-6]. A variety of stimuli have been found to induce ceramide biosynthesis in rodents, including high-fat diet [7-9] and inflammatory agonists $[8,10,11]$. Despite the wealth of research establishing the efficacy of ceramide inhibition in improving obesity-related morbidities [12], no current prescribed pharmacological therapies are known to directly inhibit ceramide biosynthesis. A potentially useful focus in exploring treatments to prevent excessive ceramide accumulation, and block ceramide-mediated pathologies related to obesity, is to identify anti-ceramide effects of currently used drugs [7]. A promising possibility is to explore the numerous drugs prescribed to treat obesity-related 
diseases that are known to regulate AMP-activated protein kinase (AMPK).

Research in recent decades has elucidated the role of the prototypical metabolic regulator AMPK in ameliorating deleterious conditions associated with obesity. Indeed, AMPK-activating drugs have been shown to improve insulin resistance $[13,14]$, cardiovascular complications $[15,16]$, and hepatic steatosis [17], which may explain their widespread use. Interestingly, many of the benefits attributed to AMPK activation are similar to those seen with ceramide inhibition $[4,6,18]$.

The metabolic functions of AMPK are increasingly clear-increased glucose uptake, increased fatty acid uptake and oxidation, and the inhibition of anabolic processes (e.g., lipogenesis) [13]. We have previously shown that AMPK inhibits inflammatory activity by blocking the NF- $\kappa B$ pathway [19], which, in light of our recent observation that ceramide biosynthesis requires inflammatory signals [8], suggests a potential ceramide-specific effect of AMPK activation. Given the general oxidative effect of AMPK on fatty acids, we sought to test the hypothesis that AMPK inhibits ceramide biosynthesis in muscle. Positive results will reveal a novel mechanism to partly explain the beneficial effects of AMPK-activating drugs in treating several obesity-related pathologies.

\section{Methods}

\section{Cell culture}

Cells were maintained in DMEM + 10\% fetal bovine serum (Invitrogen). For differentiation into myotubes, C2C12 myoblasts were grown to confluency and the medium was replaced with DMEM + 10\% horse serum (Invitrogen). Myotubes were used for experiments on day 4 of differentiation. For lipid treatment, palmitic acid (Sigma P5585) was dissolved in ethanol and diluted to desired concentration in DMEM. Palmitate solutions were then conjugated to $2 \%$ (w/v) BSA (Sigma A9576). Cells were monitored for toxicity to the high palmitic acid concentration $(0.75 \mathrm{mM})$ and no difference was observed between sample protein content. 5-amino-1- $\beta$-D-ribofuranosyl-imidazole4-carboxamide (AICAR) (Sigma A9978) was reconstituted in DMEM. Compound C (Sigma P5499) was reconstituted in DMSO.

\section{Animal study}

Male Wistar rats were kept in a temperature-controlled room with a 12:12-h light-dark cycle. Rats were fed rodent laboratory chow (8604 Harlan Teklad Rodent Diet) and water ad libitum. Treatments lasted $6 \mathrm{wk}$. During this time, rats were randomly sorted into one of four groups: no intervention (control diet; 8604 Harlan Teklad) $(n=9)$, daily AICAR injections $(n=9)$, high-fat diet (HFD) $(n=11)$, or HFD with daily AICAR injections $(n=10)$. The HFD used in this study was composed of the following ( $\mathrm{g} / \mathrm{kg}$ of food):
116.6 g olive oil, 232.7 g flax seed oil, 87.2 g sugar, 174.6 starch, 226.6 g casein, 4.5 g methionine, 30.7 g gelatin, 51.2 $\mathrm{g}$ wheat bran, $22.5 \mathrm{~g}$ vitamin mix (Harlan Teklad, AIN76A), 52.2. g mineral mix (Harlan Teklad, AIN76), 1.4 g choline chloride. The HFD was $60 \%$ fat $(40 \%$ flax seed, $20 \%$ olive oil). AICAR injections (dissolved in $0.9 \% \mathrm{NaCl}$ ) were administered subcutaneously at a dose of $0.5 \mathrm{mg} / \mathrm{g}$ body weight each morning. To reduce confounding factors, rats in the control and HFD groups were similarly handled each morning and injected with similar volumes of saline alone. All experimental procedures involving animals were approved by and performed in compliance with the Institutional Animal Care and Use Committee of Brigham Young University.

\section{Lipid analysis}

For isolation of lipids, cell and tissue pellets were resuspended in $900 \mu \mathrm{l}$ ice-cold chloroform:methanol (1:2) and incubated for $15 \mathrm{~min}$ on ice then briefly vortexed. A portion of the suspension (prior to pelleting) was used for protein measurement (Thermo Scientific). Separation of aqueous and organic phases required addition of $400 \mu \mathrm{l}$ of ice-cold water and $300 \mu \mathrm{l}$ of ice-cold chloroform. The organic phase was collected into a fresh vial and lipids were dried under a gentle nitrogen stream. An Agilent high performance liquid chromatography (HPLC) system coupled with an Applied Biosystems Triple Quadrupole/ Ion Trap mass spectrometer (3200 Qtrap) was used for quantification of individual phospholipids. Multiple reaction monitoring (MRM) transitions were set up for quantitative analysis of various polar lipids [20,21]. Levels of individual lipids were quantified using spiked internal standards, including dimyristoyl phosphatidylcholine (28:0-PC), C17-ceramide, C8-glucosylceramide, which were obtained from Avanti Polar Lipids (Alabaster, AL, USA). Neutral lipids were analyzed using a sensitivity HPLC/ESI/MRM method, modified from a previous method [22]. TAG was calculated as relative contents to the spiked d5-TAG 48:0 internal standard (CDN isotopes), while DAG was quantified using 4ME 16:0 Diether DG (Avanti) as an internal standard.

\section{Quantitative real-time PCR}

Total RNA was extracted and purified from tissues using TRIzol (Invitrogen) according to the manufacturer's recommendations. cDNA was synthesized from mRNA by reverse transcription using a commercial cDNA synthesis kit with oligo(dT) primers (iScript Select cDNA Synthesis; Bio-rad). Quantitative real-time PCR was performed by following the instructions accompanying the Qiagen Quantifast SYBER Green PCR kit and using a Qiagen QIAcube. A sample containing no cDNA was used as a non-template control to verify the absence of primer dimers. $\beta$-actin reactions were performed side by side with every sample analyzed. 
Changes in mRNA level of each gene for each treatment were normalized to that of the $\beta$-actin control mRNA according to Pfaffle [23]. Sequences are included in Table 1.

\section{Protein analysis}

Gastrocnemius was extracted from euthanized mice and flash frozen then stored at -80 . Tissues were later lysed and protein content was determined using a BCA protein assay (Pierce, Rockford, IL, USA) and sample volumes were adjusted so that precisely $50 \mu \mathrm{g}$ of protein was loaded into each lane. After addition of sample buffer, samples were resolved by SDS-PAGE, transferred to nitrocellulose, and immunoblotted with anti-SPT2 antibody (Abcam, Cambridge, MA, USA) using methods described previously [24]. After incubation with primary antibody, blots were incubated with a horseradish peroxidase-conjugated secondary antibody. Horseradish peroxidase activity was assessed with ECL solution (Thermo Scientific, Rockford, IL, USA) and exposed to film.

\section{Statistics}

Data are presented as the mean \pm SEM. Data were compared by ANOVA with Tukey's post-hoc analysis

Table 1 Sequences for PCR primers of indicated genes of interest

\begin{tabular}{|c|c|}
\hline Gene & Sequences \\
\hline & 5'-TGGCATTGTTACCAACTGGG \\
\hline \multirow[t]{2}{*}{$\beta$-actin } & 5'-GGGTCATCTITTCACGGTTG \\
\hline & 5'-TACTCAGAGACCTCCAGCTG \\
\hline \multirow[t]{2}{*}{ SPT1 } & 5'-CACCAGGGATATGCTGTCATC \\
\hline & 5'-GGAGATGCTGAAGCGGAAC \\
\hline \multirow[t]{2}{*}{ SPT2 } & 5'-GTATGAGCTGCTGACAGGCA \\
\hline & 5'-CTGTTCTACTTGGCCTGTTG \\
\hline \multirow[t]{2}{*}{ CerS1/Lass1 } & 5'-TCATGCAGGAAGAACACGAG \\
\hline & 5'-TCTTCTCAAAAAGTTCCGAG \\
\hline \multirow[t]{2}{*}{ CerS2/Lass2 } & 5'-AGTGATGATGAAAACGAATGG \\
\hline & 5'-TGGCTGCTATTAGTCTGATG \\
\hline \multirow[t]{2}{*}{ CerS3/Lass3 } & 5'-TCAGGATAAAGTAACCCCAG \\
\hline & 5'-TGTCGTTCAGCTTGAGTGAG \\
\hline \multirow[t]{2}{*}{ CerS4/Lass4 } & 5'-AGCAGGCTTCACAGAATTTC \\
\hline & 5'-CTCCAACGCTCACGAAATTC \\
\hline \multirow[t]{2}{*}{ CerS5/Lass5 } & 5'-ATGCAGACAGAAGATGAGTG \\
\hline & 5'-GTTCGGAGCATTCAACGCTG \\
\hline \multirow[t]{2}{*}{ CerS6/Lass6 } & 5'-CTGAGTCGTGAAGACAGAGG \\
\hline & 5'-CACCGGTACCTCGGAGCGGA \\
\hline Des1 & 5'-GTTTGGGATTGATGAACAGGGGT \\
\hline Glucosylceramide & 5'-GCTCAGTACATTGCTGAAGA \\
\hline Synthase & 5'-AGTACGAACCAGAGTTTTGC \\
\hline
\end{tabular}

(Graphpad Prism; La Jolla, CA). Significance was set at $P<0.05$.

\section{Results}

AICAR selectively blocks sphingolipid accumulation in muscle cells

AMPK activation is known to increase rates of mitochondrial fatty acid oxidation [25]. Based on these observations, we anticipated a general reduction in accumulated intracellular lipids when cells were treated with palmitate and AICAR when compared with palmitate treatment alone. AICAR is a well-established AMPK activator. We found that levels of the glycerolipids triacylglycerol (TAG) and diacylglycerol (DAG) (Figure $1 \mathrm{~A}$ and $\mathrm{B}$, respectively) were significantly increased in $\mathrm{C} 2 \mathrm{C} 12$ myotubes in response to prolonged palmitate treatment. AICAR addition to palmitate incubations had no significant effect on TAG and DAG levels. Similar to these glycerolipids, palmitate induced a robust increase in ceramides and glucosylceramides (Figure $1 \mathrm{C}$ and D, respectively), which have both been implicated in mediating lipidinduced insulin resistance [26,27]. However, whereas AICAR treatment had little effect of TAG and DAG, AICAR treatment blunted ceramide and glucosylceramides accrual to such a degree that levels returned to vehicle-treated conditions (Figure $1 \mathrm{C}$ and D). Despite its well-established ability to activate AMPK, AICAR may have off-target effects. To more confidently establish the importance of AMPK in mediating the sphingolipidspecific effects of AICAR, we included compound C, a specific AMPK inhibitor. The addition of compound $C$ to palmitate and AICAR treatments completely reversed the protective effect of AICAR with ceramides (Figure 1C) and glucosylceramides (Figure 1D).

\section{AICAR prevents lipid-induced transcription of SPT2 in muscle cells}

Given our findings that AICAR inhibited the synthesis of ceramide and its downstream metabolite, glucosylceramide, we sought to determine whether AICAR alters expression of enzymes involved in de novo sphingolipid biosynthesis. In support of previous findings [8], palmitate elicited a significant increase in transcript expression of multiple enzymes, including ceramide synthase 6 (CerS6), CerS2, and, finally, serine palmitoyltransferase 2 (SPT2) (Figure 2). The observations regarding SPT2 are doubly noteworthySPT2 acts as the rate-limiting enzyme in de novo sphingolipid synthesis and it was the only enzyme whose transcript level was significantly blunted with inclusion of AICAR into the palmitate-enriched media (Figure 2). Interestingly, addition of the AMPK-inhibitor compound $\mathrm{C}$ elicited a pronounced increase in SPT2 and CerS6 transcript levels. 

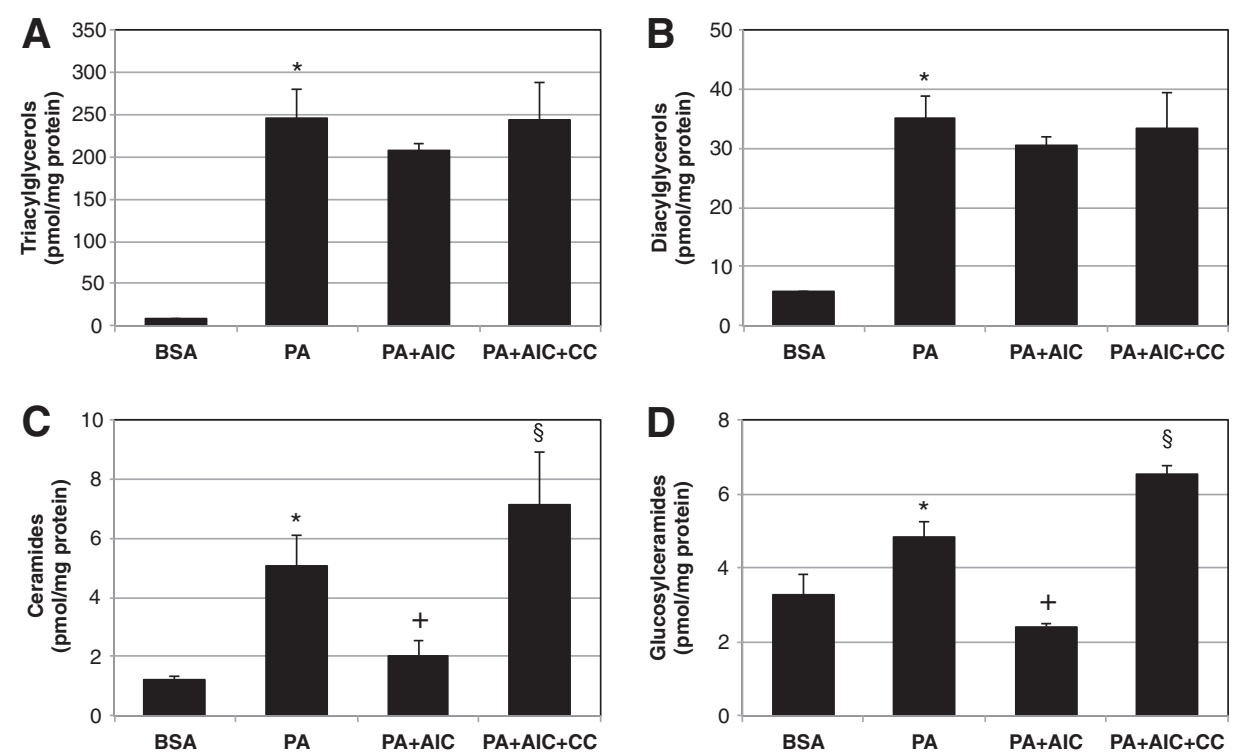

Figure 1 Palmitate-induced sphingolipid accumulation is prevented with AICAR. C2C12 myotubes were incubated with palmitate (PA) for $16 \mathrm{~h}$ at $0.75 \mathrm{mM}$. PA treatment increased all measured lipids. However, while ceramides $(\mathbf{C})$ and glucosylceramides $(\mathbf{D})$ were reduced with AICAR inclusion (2 mM), triacylglycerols (A) and diacylglycerols (B) were not significantly affected. Similarly, the inclusion of Compound C (20 $\mu M$ ) with AICAR (PA+AIC+CC) increased lipid levels over PA+AIC in ceramides $(\mathbf{C})$ and glucosylceramides (D). ${ }^{*} p<0.05$ for PA vs. BSA. $+p<0.05$ for PA+AIC vs PA. $\S p<0.05$ for $P A+A I C+C C$ vs. PA+AIC $(n=5)$.

\section{AICAR prevents high-fat diet-induced SPT2 expression}

\section{in vivo}

Having established an anti-ceramide effect of AICAR in cultured muscle cells, we next explored this relationship in whole muscle. Male Wistar rats were fed a normal chow or high-fat diet for $6 \mathrm{wk}$. During this period, animals further received daily injections of $\operatorname{AICAR}(0.5 \mathrm{mg} / \mathrm{g} \mathrm{BW})$ or vehicle (saline). Given our observations that SPT2 was

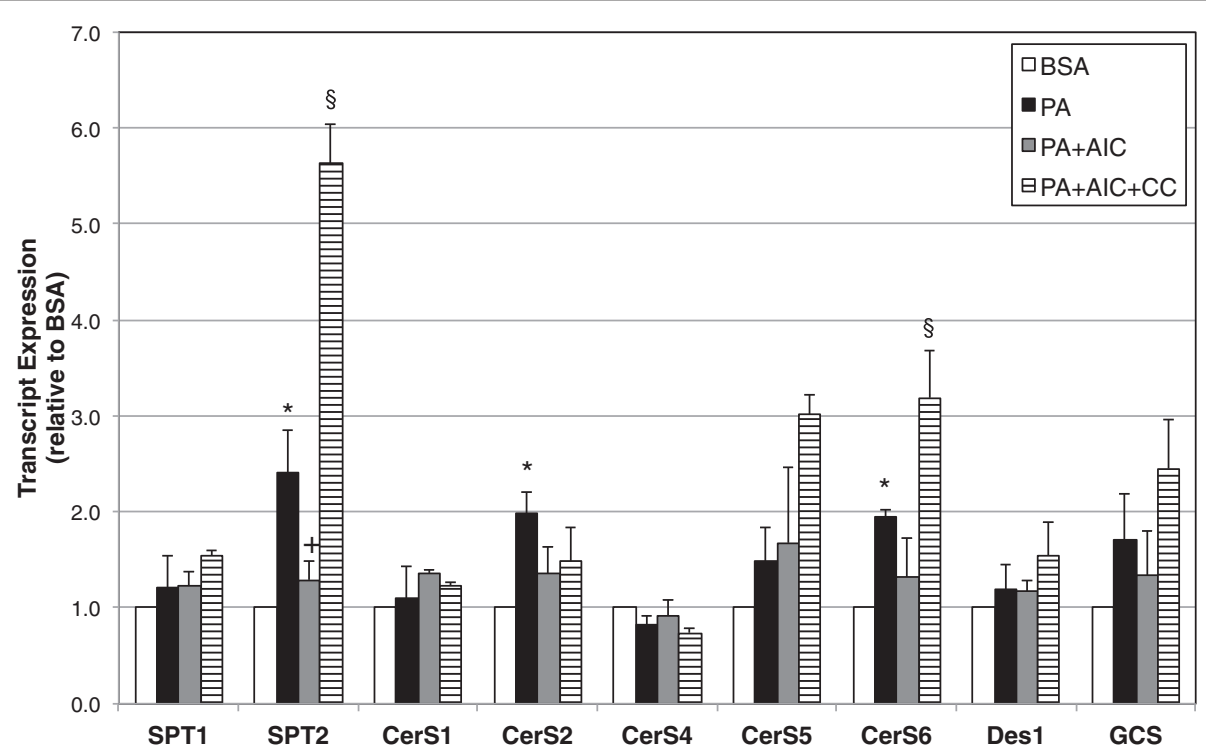

Figure 2 Palmitate increases transcription of multiple enzymes of de novo sphingolipid synthesis. $\mathrm{C} 2 \mathrm{C} 12$ myotubes were treated with palmitate (PA; 16 h, $0.75 \mathrm{mM}$ ) in the absence (PA) or presence of AICAR (PA+AIC; 2 mM). Levels of serinepalmitoyl transferase 2 (SPT2) and ceramide synthase 2 and 6 (CerS 2 and 6 ) were elevated with PA, but only SPT2 was significantly affected by inclusion of AICAR. Moreover, addition of Compound C (20 $\mu \mathrm{M})$ caused a robust increase in SPT2 and, to a lesser degree, CerS6 transcript levels. SPT1-2: serine palmitoyltransferase 1-2; CerS 1-6: ceramide synthase 1-6; Des1: dihydroceramide desaturase 1; GCS: glucosylceramide synthase. ${ }^{*} p<0.05$ for PA vs BSA. $+p<0.05$ for PA+AIC vs PA. $\$ p<0.05$ for $\mathrm{PA}+\mathrm{AIC}+\mathrm{CC}$ vs. $\mathrm{PA}+\mathrm{AIC}(\mathrm{n}=4)$. 
affected by both the lipid-laden and AICAR conditions in cells, we analyzed SPT2 expression in the muscles of rats in the control, HFD, and HFD+AICAR conditions. We found that SPT2 transcript levels increased by roughly $60 \%$ in the muscle of animals fed a HFD when compared with those receiving normal laboratory chow (Figure 3A). Importantly, prolonged AICAR injections prevented this effect, with SPT2 levels falling slightly lower than control conditions (Figure 3A). These findings were further supported by similar results with SPT2 protein levels (Figure 3B). However, the increased SPT2 transcription was insufficient to significantly increase muscle ceramide levels in rats on HFD compared with normal chow, which only tended to increase ( $p=0.068$ ) (Figure 4). Nevertheless, ceramide content from muscles of rats fed a HFD while receiving AICAR injections was significantly lower than those on HFD alone (Figure 4).

\section{Discussion}

The purpose of this study was to elucidate the targeted effect of AICAR on inhibiting de novo ceramide biosynthesis in skeletal muscle. Our data suggest that AICAR treatment, which is known to potently activate AMPK, inhibits ceramide accumulation in lipid-laden conditions by inhibiting lipid-induced expression of the rate-limiting enzyme SPT2. These findings were particularly robust in murine myotubes, which showed a dramatic increase in SPT2 expression and ceramides with palmitate exposure and a complete reversal of both trends with AICAR inclusion. Additionally, and suggestive of the importance of

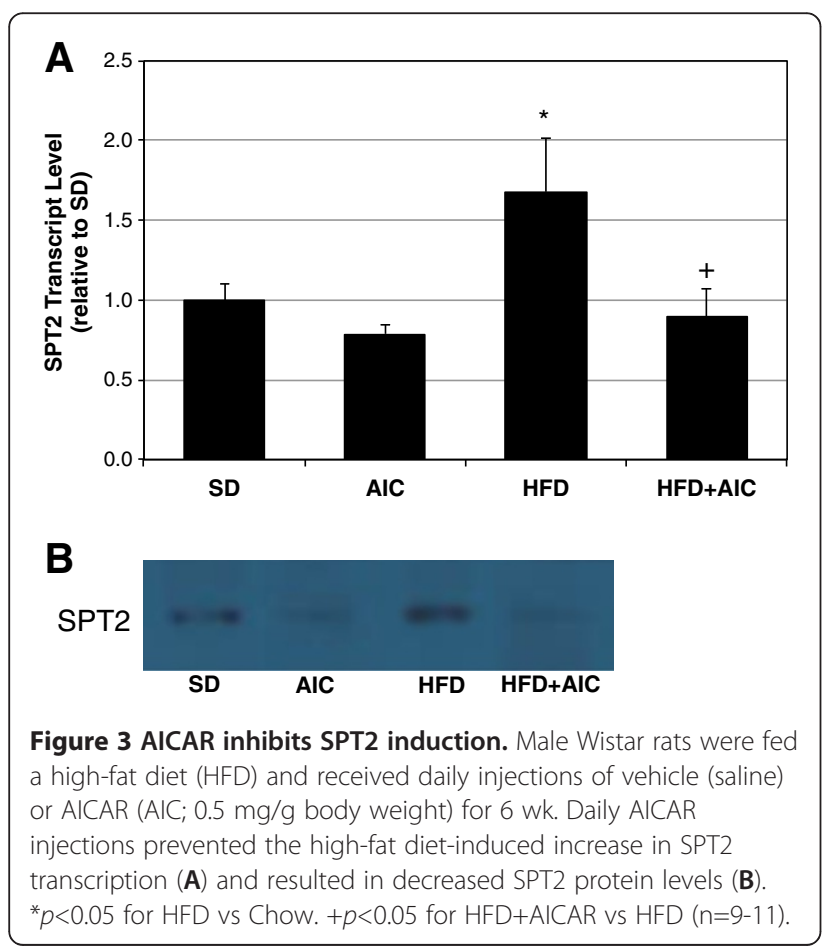

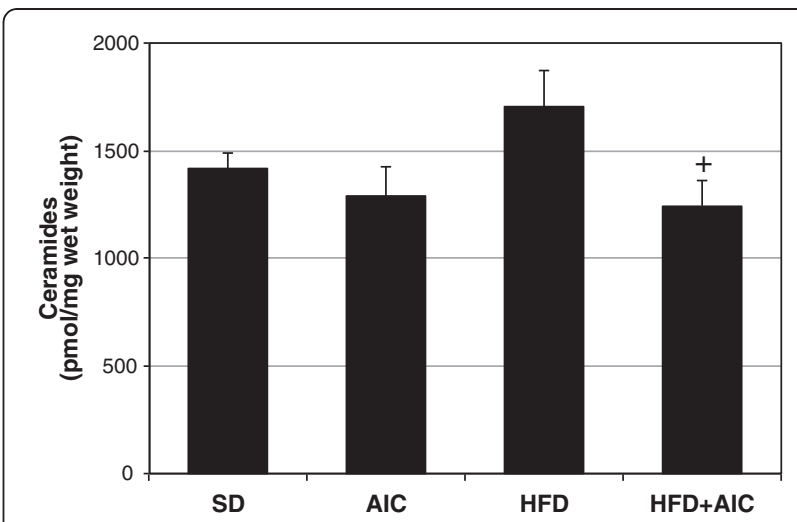

Figure 4 AICAR prevents high-fat diet-induced ceramide accumulation in muscle. Male Wistar rats were fed a high-fat diet (HFD) and received daily injections of vehicle (saline) or AICAR (AIC; $0.5 \mathrm{mg} / \mathrm{g}$ body weight) for $6 \mathrm{wk}$. HFD alone did not significantly increase ceramides compared with the Chow-fed group, but HFD+AIC resulted in a significant reduction in ceramides compared with HFD alone. $+p<0.05$ for HFD+AICAR vs HFD $(n=9-11)$.

AMPK, the addition of the AMPK inhibitor compound C increased transcript levels of SPT2 (Figure 2) and both sphingolipids (Figure $1 \mathrm{C}$ and $\mathrm{D}$ ). Less dramatic were the data from muscle from HFD-fed and AICAR-treated rats. While SPT2 expression was increased in muscle from HFD-fed rats compared with those receiving normal chow and decreased in the muscle of rats receiving AICAR injection in conjunction with HFD, actual ceramides only significantly differed between the HFD and HFD plus AICAR groups, nonetheless suggesting an in vivo effect of AICAR on ceramide inhibition (Figure 4).

A likely explanation for the lack of a more robust increase in muscle ceramides in the HFD-fed group is the saturation state of the lipids in the HFD. Previous studies that have shown increased muscle ceramide in HFD-fed models have used a HFD that is largely lard based and is, subsequently, much higher in saturated fats than the HFD used in this study, which was almost completely unsaturated (flax and olive oil). Saturated fat is not only a substrate for ceramide, but it also activates the biosynthetic pathway $[8,28]$, while unsaturated fats, particularly the monounsaturated oleic acid, do not [7]. In contrast to the in vivo findings, our use of palmitate in the cell culture provided a strong induction of ceramide biosynthesis. While the concentration used is not physiological $(0.75 \mathrm{mM})$, it is widely used as a method to potently activate ceramide biosynthetic pathways.

Our findings of an anti-ceramide effect of AICAR in skeletal muscle corroborate previous observations in nonmuscle tissues. Blazquez et al. [29] found that long-term stimulation of AMPK via AICAR treatment in astrocytes inhibited SPT activity and reduced ceramides. Collectively, these data highlight a novel specificity of AMPK activation to target ceramide synthesis in various cell types. Further, 
a possible mechanism for AMPK-induced inhibition of ceramide biosynthesis is the anti-inflammatory effects of AMPK. We recently found that ceramide biosynthesis requires NF- $\mathrm{KB}$ activation [8], which is inhibited by AMPK. Cacicedo et al. [30] observed that active AMPK prevents the inflammatory effects of both saturated fatty acids (SFA) and TNF $\alpha$ in endothelial cells. In particular, they found that the SFA- and TNFo-induced 2- to 4-fold increase in NF- $\mathrm{KB}$ reporter gene expression was prevented with both AICAR treatment and constitutively active AMPK [30].

Many prominent AMPK-activating drugs like metformin and thiazolidinediones (TZD) possess anti-inflammatory activity [31,32]. Thus, given previous findings, it is not surprising that both metformin and TZD reduce ceramide accumulation [33,34], though whether the activation of AMPK is necessary for the drugs' anti-inflammatory and anti-ceramide actions is unknown.

Previous studies have tangentially explored the relationship between AMPK and ceramides in skeletal muscle. Holland et al. [35] found that adiponectin, long known to improve insulin sensitivity and activate AMPK [36], potently stimulates ceramide conversion to sphingosine 1-phosphate (S1P), which opposes several of ceramide's deleterious effects in the cell [37]. Interestingly, S1P induces AMPK activation, which explains the observation by Holland et al. [35] that ceramide activates AMPK in muscle cells. Given our findings that AMPK inhibits ceramide biosynthesis in muscle, these studies collectively suggest a self-regulating mechanism, wherein ceramide is converted to S1P, which activates AMPK and, in turn, inhibits ceramide biosynthesis.

\section{Conclusions}

In conclusion, these experiments reveal that the AMPK activator AICAR reduces expression of SPT2 in skeletal muscle and prevents ceramide biosynthesis in hyperlipidemic conditions. Given the wide range of AMPK-activating drugs currently used to mitigate obesity-induced pathologies, our findings suggest that ceramide inhibition may be an important and novel mechanism that mediates several health benefits derived from AMPK activation.

\section{Competing interests}

The authors declare no competing interests.

\section{Authors' contributions}

KAE and MES assisted with cell treatments, RT-qPCR, lipid extraction, and manuscript preparation. TSA and JTP performed lipid analysis. MJE, ESB, AEH, $M A B, B J T, M O T$ assisted with RT-qPCR and lipid extraction. $C R H$ managed the animal study and assisted with manuscript preparation. BTB was involved in experimental planning, all experiments, and manuscript preparation. All authors read and approved the final manuscript.

\section{Acknowledgements}

The authors would like to acknowledge Marjean Garowski and Connie Provost for their excellent clerical assistance, and Doug C. Berry for his technical equipment support.

\section{Author details}

${ }^{1}$ Physiology and Developmental Biology, Brigham Young University, 593 WIDB, Provo, UT 84602, USA. ${ }^{2}$ Chemistry and Biochemistry, Brigham Young University, Provo, UT 84602, USA. ${ }^{3}$ Nutrition, Dietetics, and Food Science, Brigham Young University, Provo, UT 84602, USA.

Received: 29 June 2012 Accepted: 5 November 2012

Published: 7 November 2012

\section{References}

1. Dandona P, Aljada A, Chaudhuri A, Mohanty P, Garg R: Metabolic syndrome: a comprehensive perspective based on interactions between obesity, diabetes, and inflammation. Circulation 2005, 111:1448-1454.

2. Shaw JE, Sicree RA, Zimmet PZ: Global estimates of the prevalence of diabetes for 2010 and 2030. Diabetes Res Clin Pr 2010, 87:4-14.

3. Aggarwal BB, Higuchi M: Role of ceramide in tumour necrosis factormediated apoptosis and nuclear factor-kappa B activation. Biochem Soct T 1997, 25:1166-1171.

4. Bikman BT, Summers SA: Sphingolipids and hepatic steatosis. Adv Exp Med Biol 2011, 721:87-97.

5. Holland WL, Knotts TA, Chavez JA, Wang LP, Hoehn KL, Summers SA: Lipid mediators of insulin resistance. Nutr Rev 2007, 65:S39-S46.

6. Park TS, Rosebury W, Kindt EK, Kowala MC, Panek RL: Serine palmitoyltransferase inhibitor myriocin induces the regression of atherosclerotic plaques in hyperlipidemic ApoE-deficient mice. Pharmacol Res 2008, 58:45-51.

7. Bikman BT, Guan Y, Shui G, Siddique MM, Holland WL, Kim JY, Fabrias G, Wenk MR, Summers SA: Fenretinide prevents lipid-induced insulin resistance by blocking ceramide biosynthesis. J Biol Chem 2012, doi:10.1074/jbc.M1112.359950.

8. Holland WL, Bikman BT, Wang LP, Yuguang G, Sargent KM, Bulchand S, Knotts TA, Shui G, Clegg DJ, Wenk MR, et al: Lipid-induced insulin resistance mediated by the proinflammatory receptor TLR4 requires saturated fatty acid-induced ceramide biosynthesis in mice. J Clin Invest 2011, 121:1858-1870.

9. Blachnio-Zabielska A, Baranowski M, Zabielski P, Gorski J: Effect of high fat diet enriched with unsaturated and diet rich in saturated fatty acids on sphingolipid metabolism in rat skeletal muscle. J Cell Physiol 2010, 225:786-791.

10. Barber SA, Perera PY, Vogel SN: Defective ceramide response in $\mathrm{C} 3 \mathrm{H} / \mathrm{HeJ}$ (Lpsd) macrophages. J Immunol 1995, 155:2303-2305.

11. MacKichan ML, DeFranco AL: Role of ceramide in lipopolysaccharide (LPS)-induced signaling. LPS increases ceramide rather than acting as a structural homolog. J Biol Chem 1999, 274:1767-1775.

12. Bikman BT, Summers SA: Ceramides as modulators of cellular and wholebody metabolism. J Clin Invest 2011, 121:4222-4230.

13. Winder WW, Hardie DG: AMP-activated protein kinase, a metabolic master switch: possible roles in type 2 diabetes. Am J Physiol 1999, 277:E1-10.

14. Cleasby ME, Dzamko N, Hegarty BD, Cooney GJ, Kraegen EW, Ye JM: Metformin prevents the development of acute lipid-induced insulin resistance in the rat through altered hepatic signaling mechanisms. Diabetes 2004, 53:3258-3266.

15. Stocker DJ, Taylor AJ, Langley RW, Jezior MR, Vigersky RA: A randomized trial of the effects of rosiglitazone and metformin on inflammation and subclinical atherosclerosis in patients with type 2 diabetes. Am Heart J 2007, 153:445. e441-446.

16. Marquie G: Metformin action on lipid metabolism in lesions of experimental aortic atherosclerosis of rabbits. Atherosclerosis 1983, 47:7-17.

17. Lin HZ, Yang SQ, Chuckaree C, Kuhajda F, Ronnet G, Diehl AM: Metformin reverses fatty liver disease in obese, leptin-deficient mice. Nat Med 2000, 6:998-1003.

18. Summers SA: Ceramides in insulin resistance and lipotoxicity. Prog Lipid Res 2006, 45:42-72. 
19. Bikman BT, Zheng D, Reed MA, Hickner RC, Houmard JA, Dohm GL: Lipid-induced insulin resistance is prevented in lean and obese myotubes by AICAR treatment. Am J Physiol Regul Integr Comp Physiol 2010, 298:R1692-1699.

20. Fei W, Shui G, Gaeta B, Du X, Kuerschner L, Li P, Brown AJ, Wenk MR, Parton $R G$, Yang H: Fld1p, a functional homologue of human seipin, regulates the size of lipid droplets in yeast. J Cell Biol 2008, 180:473-482.

21. Chan R, Uchil PD, Jin J, Shui G, Ott DE, Mothes W, Wenk MR: Retroviruses human immunodeficiency virus and murine leukemia virus are enriched in phosphoinositides. J Virol 2008, 82:11228-11238.

22. Shui G, Guan X, Low CP, Chua GH, Goh SYJ, Yang H, Wenk MR: Toward one step analysis of cellular dynamic lipidome using liquid chromatography coupled with mass spectrometry: application to saccharomyces cerevisiae and schizosaccharomyces pombe lipidomics. Mol Biosyst 2010, doi:10.1039/B913353D.

23. Pfaffl MW: A new mathematical model for relative quantification in realtime RT-PCR. Nucleic Acids Res 2001, 29:e45.

24. Bikman BT, Zheng D, Pories WJ, Chapman W, Pender JR, Bowden RC, Reed MA, Cortright RN, Tapscott EB, Houmard JA, et al: Mechanism for improved insulin sensitivity after gastric bypass surgery. I Clin Endocrinol Metab 2008, 93:4656-4663.

25. Winder WW, Holmes BF, Rubink DS, Jensen EB, Chen M, Holloszy JO: Activation of AMP-activated protein kinase increases mitochondrial enzymes in skeletal muscle. J Appl Physiol 2000, 88:2219-2226.

26. Tagami S, Inokuchi Ji J, Kabayama K, Yoshimura H, Kitamura F, Uemura S, Ogawa C, Ishii A, Saito M, Ohtsuka Y, et al: Ganglioside GM3 participates in the pathological conditions of insulin resistance. J Biol Chem 2002, 277:3085-3092.

27. Bikman BT: A role for sphingolipids in the pathophysiology of obesityinduced inflammation. Cell Mol Life: CMLS 2012, doi:10.1007/s00018-00012 00917-00015.

28. Shi H, Kokoeva MV, Inouye K, Tzameli I, Yin H, Flier JS: TLR4 links innate immunity and fatty acid-induced insulin resistance. J Clin Invest 2006, 116:3015-3025.

29. Blazquez C, Geelen MJ, Velasco G, Guzman M: The AMP-activated protein kinase prevents ceramide synthesis de novo and apoptosis in astrocytes. FEBS Lett 2001, 489:149-153.

30. Cacicedo JM, Yagihashi N, Keaney JF Jr, Ruderman NB, Ido Y: AMPK inhibits fatty acid-induced increases in NF-kappaB transactivation in cultured human umbilical vein endothelial cells. Biochem Biophys Res Commun 2004, 324:1204-1209.

31. Bikman BT, Zheng D, Kane DA, Anderson EJ, Woodlief TL, Price JW, Dohm GL, Neufer PD, Cortright RN: Metformin improves insulin signaling in obese rats via reduced IKKbeta action in a fiber-type specific manner. Journal of obesity 2010, 2010. doi:10.1155/2010/970865.

32. Ruderman N, Prentki M: AMP kinase and malonyl-CoA: targets for therapy of the metabolic syndrome. Nat Rev Drug Discov 2004, 3:340-351.

33. Todd MK, Watt MJ, Le J, Hevener AL, Turcotte LP: Thiazolidinediones enhance skeletal muscle triacylglycerol synthesis while protecting against fatty acid-induced inflammation and insulin resistance. Am J Physiol Endocrinol Metab 2007, 292:E485-493.

34. Smith AC, Mullen KL, Junkin KA, Nickerson J, Chabowski A, Bonen A, Dyck

DJ: Metformin and exercise reduce muscle FAT/CD36 and lipid accumulation and blunt the progression of high-fat diet-induced hyperglycemia. Am J Physiol Endocrinol Metab 2007, 293:E172-181.

35. Holland WL, Miller RA, Wang ZV, Sun K, Barth BM, Bui HH, Davis KE, Bikman BT, Halberg N, Rutkowski JM, et al: Receptor-mediated activation of ceramidase activity initiates the pleiotropic actions of adiponectin. Nat Med 2011, 17:55-63.

36. Kadowaki T, Yamauchi T, Kubota N, Hara K, Ueki K, Tobe K: Adiponectin and adiponectin receptors in insulin resistance, diabetes, and the metabolic syndrome. J Clin Invest 2006, 116:1784-1792.

37. Takabe K, Paugh SW, Milstien S, Spiegel S: "Inside-out" signaling of sphingosine-1-phosphate: therapeutic targets. Pharmacol Rev 2008, 60:181-195.

doi:10.1186/1758-5996-4-45

Cite this article as: Erickson et al:: AICAR inhibits ceramide biosynthesis in skeletal muscle. Diabetology \& Metabolic Syndrome 2012 4:45.

\section{Submit your next manuscript to BioMed Central and take full advantage of:}

- Convenient online submission

- Thorough peer review

- No space constraints or color figure charges

- Immediate publication on acceptance

- Inclusion in PubMed, CAS, Scopus and Google Scholar

- Research which is freely available for redistribution 